\title{
Effection of UV-LED Illuminant on the Curation of Photolatent-amidine Mixed with Bisphenol A Epoxy Acrylate under Printing Conditions
}

\author{
Huawei DUAN ${ }^{1, \text { a }}$, Fanxin $\mathrm{ZENG}^{2, \mathrm{~b}}$ and Qiang $\mathrm{ZHU}^{3, \mathrm{c}}$ \\ ${ }^{1}$ Guangdong Zhuang-Li Color Printing Co.,Ltd, Chaoshan Road, Shantou, P.R.China \\ ${ }^{2,3}$ Ya-Li ECO Pcakaging Co,Ltd, Hepu Industrial Park, Shantou, P.R.China \\ a751185209@qq.com, b15768208007@163.com, ${ }^{\mathrm{c}} 870600749 @$ qq.com
}

\begin{abstract}
In order to make photocuring ink or coating cured by UV-LED illuminant, we synthesized (4-((hexahydropyrrolo[1,2-a]pyrimidin-1

(2H)-yl)methyl)phenyl)(phenyl)methanone(PL $\quad-D B N) \quad$ and (4-((octahydropyrimido[1,2-a]azepin-1(2H)-yl)methyl)phenyl)(phenyl)met hanone(PL -DBU) as photoinitiators. Different mass fraction of the photoinitiators was mixed with bisphenol A epoxy acrylate, cured by different wavelengths and power of UV-LED illuminant,and investigated the curing effects of photoinitiators on bisphenol A epoxy acrylate. The results show that the conversion of $\mathrm{C}=\mathrm{C}$ double bonds of bisphenol $\mathrm{A}$ epoxy acrylate will increase as the mass fraction of the photoinitiators or the power of UV-LED illuminant increase. In the same conditions, the curing effect of using $365 \mathrm{~nm}$ UV-LED illuminant on bisphenol A epoxy acrylate is better than using $395 \mathrm{~nm}$ UV-LED illuminant. PL-DBU has a better curing effect on the bisphenol A epoxy acrylate rather than PL-DBN. When using the $8 \mathrm{~W} / \mathrm{cm}^{2}$ of $365 \mathrm{~nm}$ UV-LED illuminant to cure a mixture of 3\% PL-DBU and $97 \%$ bisphenol A epoxy acrylate, it will be dry completely in 2 seconds, moreover, the conversion reach $79 \%$.
\end{abstract}

Keywords:(4-((hexahydropyrrolo[1,2-a]pyrimidin-1(2H)-yl)methyl)pheny 1)(phenyl)methanone; UV- illuminants; (4-((octahydropyrimido[1,2-a]azepin-1(2H)-yl)methyl)phenyl)(phenyl)met hanone; photolatent-amidine; photoinitiators

\section{Introduction}

Photolatent bases are the new photoinitiators that can generate nitrogenous bases active substance after ultraviolet(UV) irradiation. Photolatent bases are not affected by oxygen inhibition and moisture, and widely used for UV curing. It has a very broad application prospects and become a hot research photoinitiator ${ }^{[1-3]}$. Photoletent bases consist of chromophores and tertiary amine precursors. Chromophores are part of the photolatent bases, which absorb photon energy from UV rays. Tertiary amine precursors are nitrogenous bases active substance that play a part in catalytic polymerization of unsaturated resins. Kutal synthesized in the history of the first photolatent bases, which is the cobalt ammonia complex, which can generate nit-rogenous bases active substance that was used as a 
crosslinking agent in the curing of epoxy $\operatorname{resin}^{[4,5]}$. Liu introduced four kinds of chromophores on secondary nitrogen atom of octahydropyrrolo[1,2-a]pyrimidine and decahydropyrimido[1,2-a]azepine,synthesized eight photolatent-amidine with photochemical activity, and research the properties of free-radical photopolymerizations and base catalysis of them ${ }^{[6]}$. UV-LED illuminants have excellent environmental protection and energy saving property,gradually replace the traditional high pressure UV mercury lamp. UV-LED illuminants have a single radiative peak normally,and the irradiation intensity of UV-LED illuminants is weak ${ }^{[7]}$. Therefore, the curing speed of the normal UV photocureable coating and UV curable inks is slow, and the curable thickness of them is small. As a result, the normal photoinitiators can't meet the industrial production requirements.

According to reducing dicyclic amidine, and then two photolatent-amidine photoinitiators were obtained by nucleophilic substitution reation, which are (4-((hexahydropyrrolo[1,2-a]pyrimidin-1(2H)-yl)methyl)phenyl)(phenyl)methanone(PL-D $\mathrm{BN})$ and (4-((octahydropyrimido[1,2-a]azepin-1(2H)-yl)methyl)phenyl)(phenyl)methanone(PL-DBU ). In order to investigate the curing effect of the photoinitiators on bisphenol A epoxy acryl-ate resin in UV-LED illuminant, we selected different mass fraction of the photoinitiators to mix with bis-phenol A epoxy acrylate resin. The mixture was cured by different wavelengths and power of UV-LED lamps.

\section{Experimental Section}

\subsection{Experimental Materials and Instruments.}

The main experimental materials listed in table1, and the main experimental instruments listed in Tab2.

TABLE1. INFORMATION OF EXPERIMENTAL MATERIALS

\begin{tabular}{lccc}
\hline \multicolumn{1}{c}{ Raw Materials } & Mark & Purity & Provider \\
\hline $\begin{array}{l}\text { 1,5-diazabicyclo[4.3.0]non- } \\
\text { 5-ene }\end{array}$ & - & AR & Shandong Wanbo Chemical \\
1,8-diazabicyclo[5.4.0]unde & - & AR & Reagent Co,Ltd \\
c-7-ene & & & Shandong Wanbo Chemical \\
4-methylbenzophenone & - & AR & Reagent Co,Ltd \\
benzoyl peroxide & - & AR & Xiya Reagent \\
N-bromobutanimide & - & AR & Xiya Reagent \\
lithium aluminum hydride & - & AR & Xiya Reagent \\
tetrahydrofuran & - & AR & Tianjin Kemiou Chemical Reagent \\
& & & Co,Ltd \\
bisphenol A epoxy acrylate & $9105 B-$ & $20 \%$ TPG & Jiangmen Hengguang New \\
& 80 & DA & materials Co,Ltd \\
\hline
\end{tabular}


TABLE2. INFORMATION OF EXPERIMENTAL INSTRUMENTS

\begin{tabular}{ccc}
\hline Instruments & Model & Provider \\
\hline FTIR Sepctrometer & FTIR-8400 & Shimadzu \\
UV-LED & $365 \mathrm{~nm} / 395 \mathrm{n}$ & Shenzhen Weihailixin Technology development \\
illuminants & $\mathrm{m}$ & co.,LTD \\
Printability tester & $\mathrm{C} 1$ & IGT Testing Systems \\
\hline
\end{tabular}

\subsection{Organic Synthesis.}

0.1 mol 1,5-diazabicyclo[4.3.0]non-5-ene and $150 \mathrm{~mL}$ tetrahydrofuran were added to a $500 \mathrm{ml} \mathrm{3-neck} \mathrm{round} \mathrm{bottom} \mathrm{flask,} \mathrm{and} \mathrm{then} \mathrm{the} \mathrm{mixture} \mathrm{was} \mathrm{heated} \mathrm{under} \mathrm{relux} \mathrm{for} 2 \mathrm{~h}$ in the temperature range $90^{\circ} \mathrm{C}$ to $100^{\circ} \mathrm{C}$. $0.1 \mathrm{~mol}$ Lithium aluminum hydride was added to the flask step by step during the heating period. Whereafter,the mixture was stirred for $24 \mathrm{~h}$ at room temperature. Then the mixture was cooled to $8^{\circ} \mathrm{C}$ in an ice bath. We added $10 \mathrm{ml}$ saturated solution of ammonia chloride to the mixture, filtered out the sediment, removed the moisture by using sodium sulfate, at last, evaporated the organic solvent. As a result, octahydropyrrolo[1,2-a]pyrimidine(RDBN) was obtained.

$0.1 \mathrm{~mol}$ 1,8-diazabicyclo[5.4.0] undec-7-ene and $150 \mathrm{~mL}$ tetrahydrofuran were added to a $500 \mathrm{ml} 3$-neck round bottom flask, and then the mixture was heated under relux for $2 \mathrm{~h}$ in the temperature range $100^{\circ} \mathrm{C}$ to $110^{\circ} \mathrm{C} .0 .1 \mathrm{~mol}$ Lithium aluminum hydride was added to the flask step by step during the heating period. Whereafter, the mixture was stirred for $24 \mathrm{~h}$ at room temperature. Then the mixture was cooled to $8^{\circ} \mathrm{C}$ in an ice bath. We added $10 \mathrm{ml}$ saturated solution of ammonia chloride to the mixture, filtered out the sediment, removed the moisture by using sodium sulfate, at last, evaporated the organic solvent. As a result,decahydropyrimido[1,2-a]azepine(RDBU) was obtained.

$0.12 \mathrm{~mol}$ 4-methylbenzophenone(MBP)、 $200 \mathrm{ml}$ carbon tetrachloride 、 $0.1 \mathrm{~mol}$ N-bromobutanimide(NBS) and $0.1 \mathrm{~mol}$ benzoyl peroxide(BPO) were added to a $500 \mathrm{ml}$ 3 -neck round bottom flask, and then the mixture was heated under relux for $1 \mathrm{~h}$ in the temperature range $90^{\circ} \mathrm{C}$ to $95^{\circ} \mathrm{C}$. Then we filtered the mixture after it was cooled to room temperature. Subsequently, removed carbon tetrachloride from the mixture, and added $100 \mathrm{~mL}$ alcohol to the mixture.Thereout,4-(bromomethyl)benzophenone(Br-MBP) was obtained by recrystallization.

$150 \mathrm{ml}$ methylbenzene $\quad 0.3 \mathrm{~mol} \mathrm{RDBN}$ and $0.3 \mathrm{~mol} \mathrm{Br}-\mathrm{MBP}$ were added to a $500 \mathrm{ml}$ 3 -neck round bottom flask, and then the mixture was heated under relux for $24 \mathrm{~h}$ in the temperature range $80{ }^{\circ} \mathrm{C}$ to $90^{\circ} \mathrm{C}$. After separation and purification, we finally got 4-((hexahydropyrrolo[1,2-a]pyrimidin-1(2H)-yl)methyl)phenyl)(phenyl)methanone(PL-DB $\mathrm{N})$. In a same way,we got (4-((octahydropyrimido[1,2-a]azepin-1(2H)-yl)methyl)phenyl) (phenyl)methanone(PL-DBU).

\subsection{Samples and Their Text.}

The mass fraction of $1 \%, 2 \%, 3 \%, 4 \%$ of photolatent-amidine photoinitiators were mixed with bisphenol A epoxy acrylate respectively. Stirred the mixture to make it uniform. Then 
sampled the $0.1 \mathrm{cc}$ mixture, and smeared it on the binary roller of printability tester. Opened the printability tester and printed on the copper printing paper. As a rusult,a series of $30 * 30 \mathrm{~mm}$ printed samples were obtained.

The samples were exposed to the dominant wavelength of $365 \mathrm{~nm}$ and $395 \mathrm{~nm}$ UV-LED illuminants. Intermittent infrared spectroscopy was adopted to evaluate the curing rate of the conversion of $\mathrm{C}=\mathrm{C}$ double bonds ${ }^{[8,9]}$. The $\mathrm{C}-\mathrm{H}$ bonds faces to the peak of bending vibration on the $810 \mathrm{~cm}^{-1}$ of $\mathrm{C}=\mathrm{C}$ double bonds in the spectrum, and its absorption intensity decreases as UV irradiation time increases. The conversion of $\mathrm{C}=\mathrm{C}$ double bonds was caculated according to the following equation where $\mathrm{A}_{0}$ is the absorption intensity on the $810 \mathrm{~cm}^{-1}$ of $\mathrm{C}=\mathrm{C}$ double bonds without $\mathrm{UV}$ irradiation, $\mathrm{A}_{\mathrm{t}}$ is the absorption intensity on the $810 \mathrm{~cm}^{-1}$ of $\mathrm{C}=\mathrm{C}$ double bonds after $\mathrm{UV}$ irradiation, $\mathrm{C}_{\mathrm{t}}$ is the conversion of $\mathrm{C}=\mathrm{C}$ double bonds, respectively.

$$
\mathrm{C}_{\mathrm{t}}=\frac{\mathrm{A}_{0}-\mathrm{A}_{\mathrm{t}}}{\mathrm{A}_{0}} \times 100 \%
$$

\section{Results and Discussion}

\subsection{Organic Synthesis.}

Figure1 shows the synthetic routes of the photolatent-amdine. According to reducing dicyclic amidines, we got alkalescent RDBN and RDBU. Then chromophore was introduced at secondary nitrogen atoms of RDBN and RDBU by nucleophilic substitution reaction. As a result, the photolatent-amidine were obtained. The product yields and purity of all the products show Tab3.

TAB3. THE PRODUCT YIELDS AND PURITY OF ALL THE PRODUCTS

\begin{tabular}{cccccc}
\hline Products & Br-MBP & RDBN & RDBU & PL-DBN & PL-DBU \\
\hline Products Yields (\%) & 64 & 58 & 55 & 42 & 39 \\
Purity (\%) & 99.1 & 98.3 & 97.8 & 95.2 & 93.3 \\
\hline
\end{tabular}

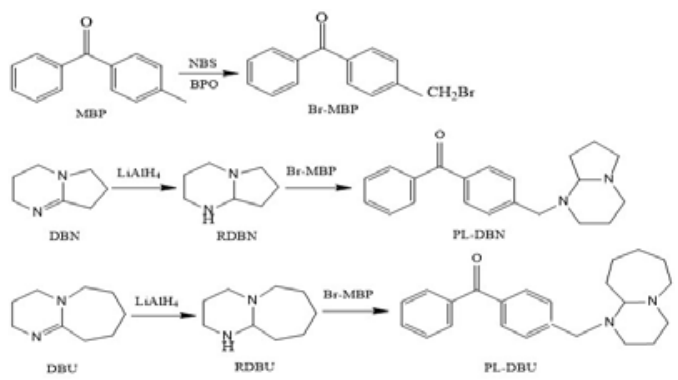

Figure1. The synthesis routes of photolatent-amidine

As shown in Figure2, photon energy excites the photolatent-amidine molecules into another excited state, the $\mathrm{C}-\mathrm{N}$ bond that chromophore connect with amidine will break and generate free radicals ${ }^{[10-12]}$. The hydrogen atom on the tertiary carbon of the amidine is 
activated by the two ortho nitrogen atoms, and then the hydrogen atom will be picked up by the chromophore free radical. Consequently, the tertiary carbon and secondary nitrogen on the amidine will combine into $\mathrm{C}=\mathrm{N}$ double bond, in other words, generate $\mathrm{DBN}$ at last. In the same principles, PL-DBU can release DBU by absorbing photon energy. Because the conjugated degree of 4-methylbenzophenone is high, correspondingly, its derivatives will lead to red shit.

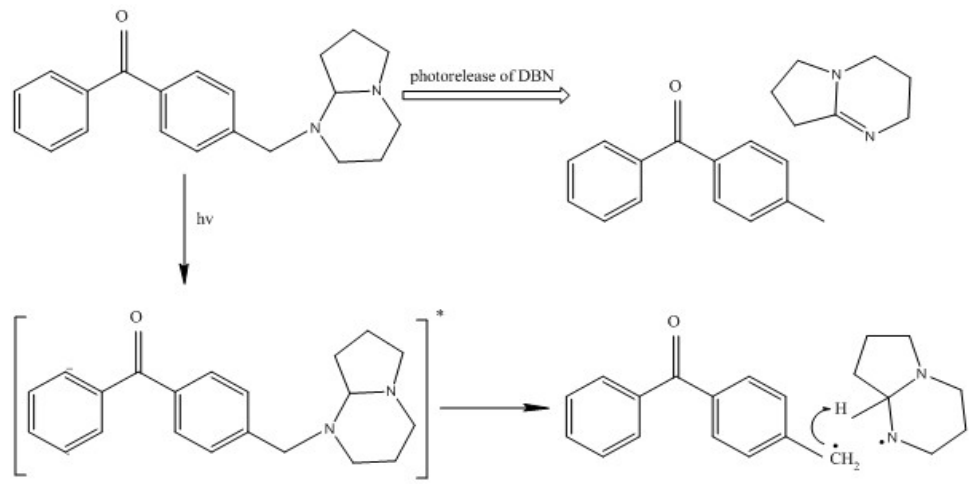

Figure2. Photochemical reaction of PL-DBN

\subsection{Mass Fraction of Photoinitiators.}

Different mass fraction of photoinitiators was added into a certain amount of bisphenol A epoxy acrylate and made into samples. The samples were exposed to $6 \mathrm{~W} / \mathrm{cm}^{2}$ of the dominant wavelength of $365 \mathrm{~nm}$ and $395 \mathrm{~nm}$ UV-LED illuminant for 3 seconds. Intermittent infrared spectroscopy was adopted to text the conversion of $\mathrm{C}=\mathrm{C}$ double bonds of the resin. The results show Figure3.

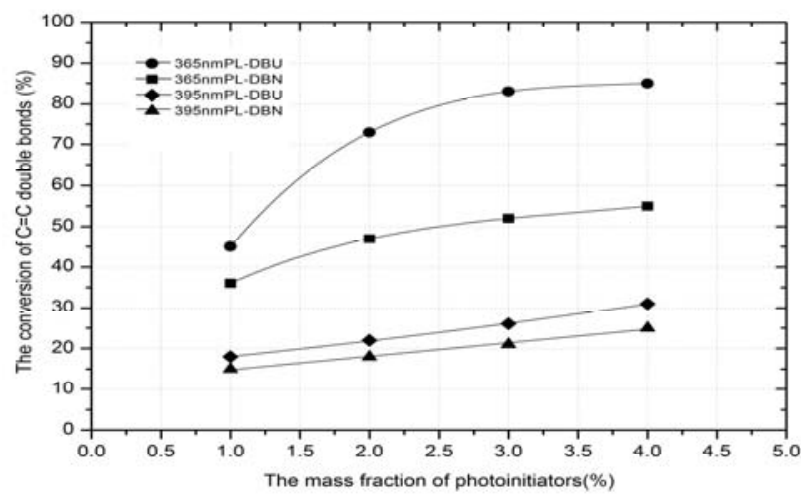

Figure3. The conversion of $\mathrm{C}=\mathrm{C}$ double bonds with different mass fraction of photoinitiators

As shown in figure 3 , the conversion of $\mathrm{C}=\mathrm{C}$ double bonds of bisphenol $\mathrm{A}$ epoxy acrylate increases as the mass fraction of photoinitiators increases in the same UV-LED illuminant. The light absorbency A is calculated according to the following equation 


$$
\mathrm{A}=\log \left(\mathrm{I}_{0} / \mathrm{I}\right)=\kappa \mathrm{c}
$$

where $\mathrm{I}_{0}$ is the intensity of the incident radiation, $\mathrm{I}$ is the intensity of transmitted radiation, $\mathrm{\kappa}$ is the molar absorption cofficient, 1 is the thickness of the sample, $\mathrm{c}$ is the mole concentration of the sample. $\kappa$ is a certain constant, and 1 is also a certain constant when the experiment conditions is same. When the mass fraction of photoinitiator increases, the mole concentration gradually become larger. It also means that the light absorbency is directly proportion to the mass fraction of photoinitiators ${ }^{[13-15]}$. Under the certain UV-LED irradiation, photoinitiators absorb less the energy of ultraviolet ray and generate less tertiary amine precursors at a lower mass fraction, as a result, the curing effect is unsatisfactory. It will be conductive to absorb the energy of ultraviolet ray and generate more tertiary amine precursors when the mass fraction of photoinitiators is increasing from $1 \%$ to $3 \%$. The surface of the resin absorb almost ultraviolet ray so that the radiant flux inside ink is few. Generating the amount of nitrogenous bases inside is directly proportion to the mass fraction of photoinitiators and the light flux. Therefore, the conversion of $\mathrm{C}=\mathrm{C}$ double bonds will flatten out after the UV-LED illuminant irradiated the samples for a period of time.

In the same conditions, the conversion of $\mathrm{C}=\mathrm{C}$ double bonds of PL-DBU/bisphenol $\mathrm{A}$ epoxy acrylate is larger than PL-DBU/bisphenol A epoxy acrylate. When the resin solidifies slowly, the ability of anti-oxygen inhibition become the main factors influencing the curing effects of the resin ${ }^{[16,17]}$. From the point of view of molecular atructure,the steric hindrance of PL-DBU is smaller than PL-DBN. It is to say that PL-DBU has the better ability of anti-oxygen inhibition, in favor of radical terimination reaction ${ }^{[18,19]}$. As a conclusion,PL-DBU is more suitable for curing bisphenol A epoxy acrylate.

\subsection{Wavelength of UV-LED Illuminant.}

As shown in Figure3, in the same conditions,365nm UV-LED illuminant is more suitable for curing PL-DBU/bisphenol A e epoxy acrylate than 395nm UV-LED illuminant. The photon energy is determined as

$$
\mathrm{E}=\frac{\mathrm{hc}}{\lambda}
$$

where $\mathrm{h}$ is Planck constant, $\mathrm{c}$ is the velocity of light, $\lambda$ is the wavelength of light. Because $\mathrm{h}$ and $c$ are certain constants, the shorter the wavelength , the higher the energy. Breaking the C-N bond that chromophore connect with amidine need to absorb the energy of ultraviolet ray. Therefore, the shorter the wavelength, the more beneficial to break the C-N bond and generate more active bases to improve curing speed and effect.

We found that the surface of the mixture could be dry completely when the conversion of $\mathrm{C}=\mathrm{C}$ double bonds of bisphenol A epoxy acrylate reached $78 \%$ in the experiment. In other words, if the conversion is above $78 \%$, it can realize dry completely. When the samples were exposed to $365 \mathrm{~nm}$ UV-LED illuminant for 3 senconds, the conversion of $\mathrm{C}=\mathrm{C}$ double bonds increase rapidly as the mass fraction of PL-DBU increases from $1 \%$ to $3 \%$ and increase slowly as the mass fraction of PL-DBU is above $3 \%$. When the sample that the mass fraction of PL-DBU is $3 \%$,exposed to the $6 \mathrm{~W} / \mathrm{cm}^{2}$ of $365 \mathrm{~nm}$ UV-LED illuminant for 3 senconds, it will realize completely dry and the conversion reach $82 \%$.

\subsection{Irradiation Intensity of UV-LED Illuminant.}

In order to investigate the influence of irradiation intensity of UV-LED illuminants on the curing effect of PL-DBU/bisphenol A epoxy acrylate, a mixture of 3\% PL-DBU and 
$97 \%$ bisphenol A epoxy acrylate is exposed to $4 \mathrm{~W} / \mathrm{cm}^{2}, 6 \mathrm{~W} / \mathrm{cm}^{2}, 8 \mathrm{~W} / \mathrm{cm}^{2}$ and $10 \mathrm{~W} / \mathrm{cm}^{2}$ of $365 \mathrm{~nm}$ UV-LED illuminants for different time,respectively. Test results were shown in Figure4.

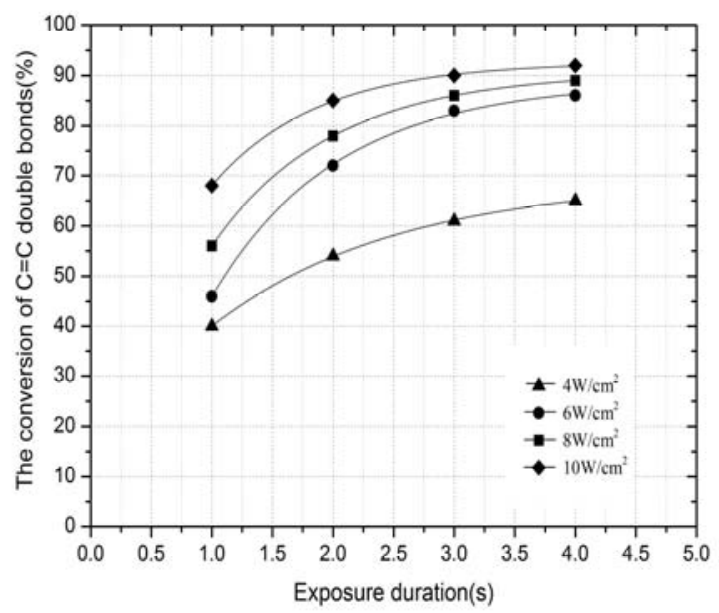

Figure4. The conversion of $\mathrm{C}=\mathrm{C}$ double bonds with different exposure duration

While the irradiation intensity of UV-LED illuminants is increasing, the conversion of $\mathrm{C}=\mathrm{C}$ double bonds of bisphenol A epoxy acrylate is increasing, and the curing speed is improving. Because more ultraviolet rays reach ink film inside, it can effectively improve the curing speed and effect. When the sample contains 3\% PL-DBU, exposed to the $8 \mathrm{~W} / \mathrm{cm}^{2}$ of $365 \mathrm{~nm}$ UV-LED illuminant for 2 senconds, it will realize completely dry and the conversion reach $79 \%$.

\section{Summary}

In the same UV-LED illuminant, the conversion of $\mathrm{C}=\mathrm{C}$ double bonds of bisphenol $\mathrm{A}$ epoxy resin acrylate increases with the mass fraction of photoinitiators. In the same conditions, the curing effect of PL-DBU on bisphenol A epoxy acrylate is better than PL-DBN, using 365nm UV-LED illuminant is more suitable for curing a mixture of PL-DBU and bisphenol A epoxy acrylate than 395nm UV-LED illuminants.Improving the irradiation intensity of UV-LED illuminants is beneficial for improving the curing speed and effect. When using the $8 \mathrm{~W} / \mathrm{cm}^{2}$ of $365 \mathrm{~nm}$ UV-LED illuminant to cure a mixture of $3 \%$ PL-DBU and 97\% bisphenol A epoxy acrylate,it will be dry completely in 2 seconds, moreover, the conversion reach $79 \%$.

\section{References}

1. Shirai M, Tsunooka M. Photobase generators:chemistry and applications to polymeric materials. Prog Polym Sci,1996,21:1-45.

2. Dietliker K, Jung T, Benkhoff J, etal. New developments in photoinitiators. Macromol Symp, 2004,217:77-97.

3. Suyama K, Shirai M. Photobase generators:recent progress and application trend inpolymer systems. Prog Polym Sci, 2009,34:194-209. 
4. Kutal C, Willson C, Grant J. Photoinitiated cross-linking and image formation in thin polymer films containing a transition metal compound. Electrochem Soc, 1987,134:2280-2285.

5. Schwalm R. UV coatings:basics, recent developments and new applications. Els-evier Publishers, 2006,56-85.

6. Liu Lei. Study on sythesis and application of photolatent-amidine photoinitiators. Guangzhou: South China University of Technology, 2012:21-30.

7. Yi Qing, Wei Xianfu, etal. Research on Photoinitiator and Illuminant of UV-LED ink. Journal of Beijing Institute of Graphic Communication, 2013,21(6):42-48.

8. Hyo-sook Joo, Young-Jun Park, Hyun-Sung Do, etal. The Curing Performance of UV-curable Semi-interpenetrating Polymer Network Structured Acrylic Pressure-sensitive Adhesives. Journal of Adhesion Science and Technoligy, 2007(7):575-588.

9. Ma Guozhang, Wu Jianbing, $\mathrm{Xu}$ Bingshe. Study on the conversion of Acrylic $\mathrm{C}=\mathrm{C}$ double bonds during Dark Reaction after UV Curing Using Infrared Spectroscopy. Spectroscopy and Spectral Analysis, 2010,30(7):1780-1784.

10. Wang Hailong. Light Curing control and evaluate the performance of its film. http://wenku.baidu.com/ view/8d78b359be23482fb4da4ca6,2013-10-05.

11. Dietliker K, Jung T,Studer K, etal. Photolatent tertiary amines-A new technology platform for radiation curing. CHIMIA, 2007,61:655-660.

12. Okamura H, Skin K, Shirai M. Photocrosslinking system using highlyfunction-alized epoxy crosslinkers having degradable property. Polymer Journal, 2006,38:1237-1277.

13. Wang Dehai, Jiang Feng. UV-curing Material : Theory and Application.Beijing:Chemical Industry Press, 2001: 30-55.

14. Studer K, Jung T, Studer K, etal. Photolatent bases:new catalysts for UV-curing of coating.PU Mag. 2006,3:2-5.

15. Christopher M.S,Mark E.N. Epoxy thiol photolatent base clearcoats:curing and formulation.J Coat Technol Res, 2010.

16. Yang Jianwen, Zeng Zhaohua, Chen Yonglie. UV-curing material and application.Beijing:Chemical Industry Press, 2005:7-9.

17. Wang Dehai, Jiang Feng. UV-curing Material : Theory and Application. Beijing:Chemical Industry Press, 2001: 71-75.

18. Jiang X.S, Yin J. Polymeric photoinitiator containing in-chain thioxanthone and oninitiator amine. Macromol Rapid Commun, 2004,25:748.

19. Jiang X.S. Study of macrophotoinitiator containing in-chain thioxanthone and coinitiator amines. Polymer,2004,45:5057. 